ginn einer neuen Epoche stehe, und er versucht damit den Blick von zu engen, detailproblembedingten Horizonten zu befreien (249-256). Ob freilich langfristig (im Hinblick auf Weltprobleme der Menschheitsernährung) die Aussicht bestehen bleiben wird, daß landwirtschaftliche Flächen für andere Zwecke frei werden, bleibt fraglich, und damit auch die Frage nach der „Landwirtschaft in der Welt von morgen" noch offener als Priebe sie sieht. - Es folgen speziellere Beiträge: Industrielle Standortwahl und regionalpolitische Implikationen (Hansmeyer/Fürst! Zimmermann, 257-288), Grundprobleme der europäischen Regionalpolitik (Jochimsen/Treuner, 289-304), Aufbau und Anwendung von Regionalmodellen (Scherer, 305-330), Regionale Auswirkungen des einzelbetrieblichen Förderungsprogramms (Reisch, 331-354), Produktionskapazität und Agrarstrukturen (Pfähler, 355-368), Überbetriebliche Zusammenarbeit und Vertragslandwirtschaft Integrationsformen in der Landwirtschaft (Werschnitzky, 369-390), Die nebenberufliche Landwirtschaft in gesamtwirtschaftlicher Sicht (Ort, 391-424).

Zur Einleitung des entwicklungspolitischen Abschnittes zieht Priebe die „Lehren aus der europäischen Wirtschafts-Integration für die Entwicklungsländer" (427446). Der Bereich, aus dem dabei die Lehren gezogen werden, beschränkt sich freilich nicht auf die jüngere europäische Agrarpolitik. Mit einerseits allgemeinen und andererseits recht speziellen Themen wird der Band beendet: Entwicklungspolitik und ökonomische Theorie (Sohn, 447-468), Anwendbarkeit gesamtwirtschaftlicher Modelle auf Entwicklungsländer (Enke, 469-488), Das Spannungsverhältnis zwischen Agrarprotektionismus und Entwicklungspolitik (Matzke, 489-515), Familienwirtschaft in Israel (Weitz, 517-538), Wirtschaftlicher Wandel und kleine Familienbetriebe in Westpakistan (Ali Khan, 539-551), Übernationale wirtschaftliche Integration in Westafrika (Bleckmann, 553-575), Zur Aufstellung von Regionalplänen in Entwicklungsländern (Urff, 576-605). - Gerade die Themen des letzten Abschnittes machen deutlich, daß der gesamte Band nicht so bald an Aktualität verlieren wird, weder innerhalb der EG, noch in der übrigen Welt.

Dieter Suhr

\title{
Handbuch Vereinte Nationen
}

Herausgegeben von Rüdiger Wolfrum, Norbert J. Prill, Jens A. Brückner Verlag Dokumentation, München 1977, 577 Seiten; DM 98,--

Das Handbuch setzt sich zum Ziel, „Strukturen, Funktionen und Arbeitsweise der Vereinten Nationen und ihrer Sonderorganisationen" darzustellen; es will Universitäten und der interessierten Öffentlichkeit Antworten auf juristische und politologische Fragen geben, die die Tätigkeit der Vereinten Nationen aufwirft. Unter 111 Stichworten präsentieren zu diesem Zweck 72 Autoren (über deren Vitae das 14seitige Autorenverzeichnis ausführlich Auskunft gibt) Artikel von zumeist vier bis acht Seiten Länge (aus dem Rahmen fallen insoweit "Nahost“ (Büttner) mit 15 Seiten, „Weltwirtschaftsordnung “ (Prill) mit 12 Seiten Länge). Einige Stichworte werden von Autoren behandelt, die zu den betreffenden Sachgebieten bereits umfangreichere Arbeiten publiziert haben (z. B. Kimminich - „Abrüstung“; Delbrück — "Apartheid“, „Diskriminierung“; Ansprenger — „Befreiungsbewegungen “, „Entkolonialisierung“; Beyerlin — „Intervention“; Bothe — „ABC-Waffen“) oder ihm durch ihre berufliche Tätigkeit nahestehen (z. B. der apostolische Nuntius Monsignore Cardinale — „Kirche und Vereinte Nationen“; der ehemalige Leiter 
des Informationsdienstes der UNO in Genf Baumgarten - „Informationswesen“; der Generalsekretär des Deutschen Roten Kreuzes Schlögel - „Internationales Rotes Kreuz").

Die folgenden Bemerkungen beziehen sich nur auf Stichwortauswahl und Benutzbarkeit des Bandes.

Die Auswahl der Einträge orientiert sich zum einen an Struktur und System der Vereinten Nationen. Die wichtigsten Institutionen der UNO selbst (unter Verzicht auf das Verwaltungsgericht), die Organisationen der sog. UNO-Familie (diese in besonders gut dokumentierten Abschnitten), ferner die Grundbegriffe der Charta (z. B. "Gewaltverbot" - Doeker; "Grundsätze der Vereinten Nationen" ders.) und die Hauptfunktion der Vereinten Nationen (z. B. „Friedenssicherung “ Blumenwitz; „Kollektive Sicherheit“ - Hütter) werden vorgestellt. Der völkerrechtliche Hintergrund dazu wird aufbereitet durch zwei völkerrechtshistorische Beiträge Hermann Webers („Entstehungsgeschichte“; „Völkerbund“) sowie einige Artikel zu allgemeinen Rechtsbegriffen (z. B. „Abkommen, Verträge“ — P. Meyer/ K. Ipsen; „Souveränität“ - Meesen; „Vertretung “ - Seidl-Hohenveldern), die jeweils unter Akzentuierung der UNO-spezifischen Besonderheiten abgefaßt sind.

Dieser systematischen Auswahl wird eine solche nach Staaten und Regionen sowie politischen Problembereichen zugesellt, die in der Praxis der Vereinten Nationen eine besondere Rolle gespielt haben (z. B. „Nigeria“ - Ansprenger; „Indischer Subkontinent" - H. W. Schulz; "Seerecht" - Wolfrum; „Rohstoffabkommen“ Wehser). Denkbar ist, daß der so untergebrachte Stoff auch nach dem eben „systematisch" genannten Auswahlprinzip hätte eingeordnet werden können; das von den Herausgebern gewählte Vorgehen ist allerdings möglicherweise zwangloser und ermöglicht eine geschlossenere Darstellung. Freilich wird die Aktualität des Handbuches auf diese Weise recht stark vom Fortgang des politischen Geschehens um die Vereinten Nationen abhängig gemacht - manche „Krise“ wird mit den Jahren ihre Bedeutung verlieren, weitere Regionen (das „Horn von Afrika“) rücken in den Mittelpunkt -, so daß eine Fortführung des Buches schon die Stichwortauswahl wird überprüfen müssen. Die Zuordnung zu einem regionalen oder einem Problemstichwort überzeugt im übrigen nicht durchweg (die Republik Südafrika hat kein eigenes Stichwort - ihr „Fall“ findet sich unter „Südliches Afrika“ (von Lucius) und „Apartheid“ (Delbrück).

Der Benutzer des Handbuches wird daher häufig unsicher sein, unter welchem Stichwort er die gesuchte Information nachschlagen soll; ein Register steht ihm dabei nicht zur Verfügung. Statt dessen haben die Herausgeber ein Verweisungssystem eingerichtet, das im Inhaltsverzeichnis zeigt, auf welche anderen Beiträge des Handbuches ein Artikel jeweils Bezug nimmt. Der Sinn dieses Systems, Zusammenhänge überblickartig zu verdeutlichen, ist freilich mitunter zweifelhaft (so sind bei „Revision der Charta“ (M. Schröder) u. a. aufgeführt: „ECOSOC; Generalsekretär; IGH; Mitgliedschaft; Sicherheitsrat; Souveränität“ — zu welchem Nutzen). Wer sich mit dem Buch erst einmal vertraut gemacht hat, sollte sich allerdings auch ohne Register zurechtfinden können.

Die Beiträge beschränken sich meist auf Bericht und Information und halten einen Mittelweg zwischen lexikonartigem und diskursivem Stil; zu Streitfragen werden selten Positionen bezogen - das Handbuch ist insofern ungleich zurückhaltender als der ja häufig meinungsbildende "Strupp/Schlochauer“. Auffällig ist die oft wenig glückliche Literaturauswahl, was bei einem Werk, das „Einstiegsmöglichkei- 
ten" vermitteln soll, besonders ins Gewicht fällt: bei "Gewaltverbot" (Doeker) fehlt der wichtige Sammelband Schaumanns von 19711; bei "Suchtstoffe“ (Boroffka) fehlt der Kommentar des Generalsekretärs zu dem Einheitsübereinkommen von $1961^{2}$ sowie die wichtige Aufsatzliteratur ${ }^{3}$, statt dessen wird auf die Encyclopedia Britannica, Stichwort „United Nations“, und einen schwer zugänglichen Bericht der WHO verwiesen; bei „Befreiungsbewegung“ (Ansprenger) werden sieben politikwissenschaftliche und eine knappe juristische Arbeit, nicht aber die eingehenden völkerrechtlichen Untersuchungen von Lazarus und von Wedel ${ }^{4}$ aufgeführt; bei „Resolution, Erklärung, Beschluß“ (Lagoni) wird die handliche Sammlung von Resolutionen der Generalversammlung, die Krakau, von Wedel und Göhmann 1975 vorgelegt haben und die gerade für die von dem Handbuch angepeilte „interessierte Öffentlichkeit" benutzbar ist, nicht erwähnt. Dankenswert ist der Anhang mit einem Wegweiser durch die UNO-Publikationen, der die von Kasme besorgte Übersicht in Sørensens Manual of Public International $\mathrm{Law}^{5}$ an Übersichtlichkeit übertrifft.

Die einleitend referierte Zielsetzung verwirklicht zu haben, kann dem Handbuch insgesamt attestiert werden. Mit der Materie wissenschaftlich befaßten Benutzern wird es selten ausreichende Antworten, fast immer aber nützliche Hinweise geben können.

Philip Kunig

\footnotetext{
1 W. Schaumann (Hrsg.), Völkerrechtliches Gewaltverbot und Friedenssicherung.

2 Commentary on the Single Convention on Narcotic Drugs, 1973.

3 Z. B. Waddell, AJIL 64 (1970), S. $310 \mathrm{ff}$.

4 AFDI 20 (1974), S. 1973 ff., bzw. RDI 1974, S. $185 \mathrm{ff}$

51968 , S. $873 \mathrm{ff}$.
} 